Supplementary Information for

\title{
Molecular Structures of Isolevuglandin-protein Cross-Links
}

By Wenzhao Bi, Geeng-Fu Jang, Lei Zhang, John W. Crabb, James Laird, Mikhail Linetsky and

Robert G. Salomon

Item

Pages

Table S1. Molecular weight summary iso[4] $\mathrm{LGE}_{2}$ peptide derivatives.

S2-S3

Figure S1. Acetyl-Gly-Lys-O-Methyl ester reactions with iso[4] $\mathrm{LGE}_{2}$ in different S4-S12 molar ratio and time points.

Figure S2. ESI-MS/MS of molecular ions at A) aminal cross-link, B) pyrrole, C) lactam and D) hydroxylactam corresponding to an iso[4] $\mathrm{LGE}_{2}-(\mathrm{N}-$ Acetyl-Gly-Lys-OMe) adduct.

Scheme S1. Possible fragmentation of (N-Acetyl-Gly-Lys-OMe)-iso[4]LGE ${ }_{2}$ S14 adducts.

Figure S3. Amyloid (11-17) EVHHQKL peptide reactions with iso[4]LGE $\mathrm{L}_{2}$ in $\mathrm{S} 15-20$ different molar ratio and time points. 
Table S1. Molecular weight summary iso[4] $\mathrm{LGE}_{2}$ peptide derivatives.

Molecular weight summary of N-Acetyl-Gly-Lys-O-Methyl Ester reaction with iso[4] $\mathrm{LGE}_{2}$ (A) N-Acetyl-Gly-Lys-O-Methyl Ester (Average molecular weight): 259.3056

H:1.008 $\mathrm{H}_{2} \mathrm{O}: 18.0153$

\begin{tabular}{|l|l|l|l|l|l|}
\hline Adducts & $\begin{array}{l}\text { Adduct } \\
\text { Formula }\end{array}$ & $\begin{array}{l}\text { Adduct } \\
\text { MW }\end{array}$ & $\begin{array}{l}\text { Product } \\
\text { MW }\end{array}$ & $\begin{array}{l}\text { Plus one } \\
\text { charge (+1) }\end{array}$ & $\begin{array}{l}\text { Plus two } \\
\text { charges (+2) }\end{array}$ \\
\hline Pyrrole & $\mathrm{C}_{20} \mathrm{H}_{28} \mathrm{O}_{3}$ & 316.4405 & 575.7461 & 576.7541 & 288.88105 \\
\hline Pyrrole $-\mathrm{H}_{2} \mathrm{O}$ & $\mathrm{C}_{20} \mathrm{H}_{26} \mathrm{O}_{2}$ & 298.4252 & 557.7308 & 558.7388 & 279.8734 \\
\hline Lactam & $\mathrm{C}_{20} \mathrm{H}_{28} \mathrm{O}_{4}$ & 332.4399 & 591.7455 & 592.7535 & 296.88075 \\
\hline Lactam- $\mathrm{H}_{2} \mathrm{O}$ & $\mathrm{C}_{20} \mathrm{H}_{26} \mathrm{O}_{3}$ & 314.4246 & 573.7302 & 574.7382 & 287.8731 \\
\hline Hydroxylactam & $\mathrm{C}_{20} \mathrm{H}_{28} \mathrm{O}_{5}$ & 348.4393 & 607.7449 & 608.7529 & 304.88045 \\
\hline Hydroxylactam- $\mathrm{H}_{2} \mathrm{O}$ & $\mathrm{C}_{20} \mathrm{H}_{26} \mathrm{O}_{4}$ & 330.4240 & 589.7296 & 590.7376 & 295.8728 \\
\hline Hydroxylactam-2 $\mathrm{H}_{2} \mathrm{O}$ & $\mathrm{C}_{20} \mathrm{H}_{24} \mathrm{O}_{3}$ & 312.4088 & 571.7144 & 572.7224 & 286.8652 \\
\hline Cross-link & $\mathrm{C}_{20} \mathrm{H}_{33} \mathrm{O}_{4}$ & 337.4796 & 853.0668 & 854.0748 & 427.5414 \\
\hline Cross-link- $\mathrm{H}_{2} \mathrm{O}$ & $\mathrm{C}_{20} \mathrm{H}_{31} \mathrm{O}_{3}$ & 319.4643 & 835.0515 & 836.0595 & 418.53375 \\
\hline Cross-link-2 $\mathrm{H}_{2} \mathrm{O}$ & $\mathrm{C}_{20} \mathrm{H}_{29} \mathrm{O}_{2}$ & 301.4491 & 817.0362 & 818.0442 & 409.5261 \\
\hline Bis-pyrrole & $\mathrm{C}_{40} \mathrm{H}_{58} \mathrm{O}_{6}$ & 634.8969 & 1149.4761 & 1150.4841 & 575.74605 \\
\hline Bis-pyrrole- $\mathrm{H}_{2} \mathrm{O}$ & $\mathrm{C}_{40} \mathrm{H}_{56} \mathrm{O}_{5}$ & 616.8816 & 1131.4608 & 1132.4688 & 566.7384 \\
\hline Bis-pyrrole- $2 \mathrm{H}_{2} \mathrm{O}$ & $\mathrm{C}_{40} \mathrm{H}_{54} \mathrm{O}_{4}$ & 598.8664 & 1113.4455 & 1114.4535 & 557.73075 \\
\hline Tris-pyrrole & $\mathrm{C}_{60} \mathrm{H}_{88} \mathrm{O}_{9}$ & 953.3353 & 1725.2041 & 1726.2121 & 863.6101 \\
\hline Tris-pyrrole- $\mathrm{H}_{2} \mathrm{O}$ & $\mathrm{C}_{60} \mathrm{H}_{86} \mathrm{O}_{8}$ & 935.3200 & 1707.1888 & 1708.1968 & 854.6024 \\
\hline Tris-pyrrole-2 $\mathrm{H}_{2} \mathrm{O}$ & $\mathrm{C}_{60} \mathrm{H}_{84} \mathrm{O}_{7}$ & 917.3047 & 1689.1735 & 1690.1815 & 845.5948 \\
\hline Tris-pyrrole- $3 \mathrm{H}_{2} \mathrm{O}$ & $\mathrm{C}_{60} \mathrm{H}_{82} \mathrm{O}_{6}$ & 899.2894 & 1671.1582 & 1672.1662 & 836.5871 \\
\hline
\end{tabular}

(B) N-Acetyl-Gly-Lys-O-Methyl Ester (Monoisotopic molec weight): 259.1532

H:1.0078 $\mathrm{H}_{2} \mathrm{O}: 18.0106$

\begin{tabular}{|l|l|l|l|l|l|}
\hline Adducts & $\begin{array}{l}\text { Adduct } \\
\text { Formula }\end{array}$ & $\begin{array}{l}\text { Adduct } \\
\text { MW }\end{array}$ & $\begin{array}{l}\text { Product } \\
\text { MW }\end{array}$ & $\begin{array}{l}\text { Plus one } \\
\text { charge (+1) }\end{array}$ & $\begin{array}{l}\text { Plus two } \\
\text { charges (+2) }\end{array}$ \\
\hline Pyrrole & $\mathrm{C}_{20} \mathrm{H}_{28} \mathrm{O}_{3}$ & 316.2038 & 575.3570 & 576.3648 & 288.6863 \\
\hline Pyrrole $-\mathrm{H}_{2} \mathrm{O}$ & $\mathrm{C}_{20} \mathrm{H}_{26} \mathrm{O}_{2}$ & 298.1933 & 557.3465 & 558.3543 & 279.68105 \\
\hline Lactam & $\mathrm{C}_{20} \mathrm{H}_{28} \mathrm{O}_{4}$ & 332.1988 & 591.3520 & 592.3598 & 296.6838 \\
\hline Lactam- $\mathrm{H}_{2} \mathrm{O}$ & $\mathrm{C}_{20} \mathrm{H}_{26} \mathrm{O}_{3}$ & 314.1882 & 573.3414 & 574.3492 & 287.6785 \\
\hline Hydroxylactam & $\mathrm{C}_{20} \mathrm{H}_{28} \mathrm{O}_{5}$ & 348.1937 & 607.3469 & 608.3547 & 304.68125 \\
\hline Hydroxylactam- $\mathrm{H}_{2} \mathrm{O}$ & $\mathrm{C}_{20} \mathrm{H}_{26} \mathrm{O}_{4}$ & 330.1831 & 589.3363 & 590.3441 & 295.67595 \\
\hline Hydroxylactam-2 $\mathrm{H}_{2} \mathrm{O}$ & $\mathrm{C}_{20} \mathrm{H}_{24} \mathrm{O}_{3}$ & 312.1725 & 571.3257 & 572.3335 & 286.67065 \\
\hline Cross-link & $\mathrm{C}_{20} \mathrm{H}_{33} \mathrm{O}_{4}$ & 337.2379 & 852.5209 & 853.5287 & 427.26825 \\
\hline Cross-link- $\mathrm{H}_{2} \mathrm{O}$ & $\mathrm{C}_{20} \mathrm{H}_{31} \mathrm{O}_{3}$ & 319.2273 & 834.5103 & 835.5181 & 418.26295 \\
\hline Cross-link-2H $\mathrm{H}_{2} \mathrm{O}$ & $\mathrm{C}_{20} \mathrm{H}_{29} \mathrm{O}_{2}$ & 301.2168 & 816.4997 & 817.5075 & 409.25765 \\
\hline Bis-pyrrole & $\mathrm{C}_{40} \mathrm{H}_{58} \mathrm{O}_{6}$ & 634.4233 & 1148.6985 & 1149.7063 & 575.35705 \\
\hline Bis-pyrrole- $\mathrm{H}_{2} \mathrm{O}$ & $\mathrm{C}_{40} \mathrm{H}_{56} \mathrm{O}_{5}$ & 616.4128 & 1130.6879 & 1131.6957 & 566.35175 \\
\hline Bis-pyrrole-2 $2 \mathrm{H}_{2} \mathrm{O}$ & $\mathrm{C}_{40} \mathrm{H}_{54} \mathrm{O}_{4}$ & 598.4022 & 1112.6773 & 1113.6851 & 557.34645 \\
\hline Tris-pyrrole & $\mathrm{C}_{60} \mathrm{H}_{88} \mathrm{O}_{9}$ & 952.6428 & 1724.0556 & 1725.0634 & 863.0356 \\
\hline Tris-pyrrole- $\mathrm{H}_{2} \mathrm{O}$ & $\mathrm{C}_{60} \mathrm{H}_{86} \mathrm{O}_{8}$ & 934.6322 & 1706.0450 & 1707.0528 & 854.0303 \\
\hline Tris-pyrrole-2 $\mathrm{H}_{2} \mathrm{O}$ & $\mathrm{C}_{60} \mathrm{H}_{84} \mathrm{O}_{7}$ & 916.6216 & 1688.0344 & 1689.0422 & 845.0250 \\
\hline Tris-pyrrole- $3 \mathrm{H}_{2} \mathrm{O}$ & $\mathrm{C}_{60} \mathrm{H}_{82} \mathrm{O}_{6}$ & 898.6110 & 1670.0238 & 1671.0316 & 836.0197 \\
\hline
\end{tabular}


Molecular weight summary of Amyloid (11-17) "EVHHQKL" peptide reaction with iso[4]LGE 2 EVHHQKL (Average molec wt): 889.99854, (+1) 891.00593, $(+2) 446.0666 \quad \mathrm{H}: 1.008 \quad \mathrm{H}_{2} \mathrm{O}: 18.0153$

\begin{tabular}{|c|c|c|c|c|c|c|c|}
\hline Adducts & $\begin{array}{l}\text { Adduct } \\
\text { Formula }\end{array}$ & $\begin{array}{l}\text { Adduct } \\
\text { MW }\end{array}$ & $\begin{array}{l}\text { Product } \\
\text { MW }\end{array}$ & $\begin{array}{l}\begin{array}{l}\text { Plus one } \\
\text { charge }(+1)\end{array} \\
\end{array}$ & $\begin{array}{l}\text { Plus two } \\
\text { charges }(+2)\end{array}$ & $\begin{array}{l}\text { Plus three } \\
\text { charges }(+3)\end{array}$ & $\begin{array}{l}\begin{array}{l}\text { Plus four } \\
\text { charges }(+4)\end{array} \\
\end{array}$ \\
\hline Pyrrole & $\mathrm{C}_{20} \mathrm{H}_{28} \mathrm{O}_{3}$ & 316.4405 & 1206.4390 & 1207.4470 & 604.2275 & 403.15433 & 302.61775 \\
\hline Pyrrole $-\mathrm{H}_{2} \mathrm{O}$ & $\mathrm{C}_{20} \mathrm{H}_{26} \mathrm{O}_{2}$ & 298.4252 & 1188.4237 & 1189.4317 & 595.21985 & 397.14923 & 298.11392 \\
\hline Lactam & $\mathrm{C}_{20} \mathrm{H}_{28} \mathrm{O}_{4}$ & 332.4399 & 1222.4384 & 1223.4464 & 612.2272 & 408.48746 & 306.6176 \\
\hline Lactam- $\mathrm{H}_{2} \mathrm{O}$ & $\mathrm{C}_{20} \mathrm{H}_{26} \mathrm{O}_{3}$ & 314.4246 & 1204.4231 & 1205.4311 & 603.21955 & 402.48236 & 302.11377 \\
\hline Hydroxylactam & $\mathrm{C}_{20} \mathrm{H}_{28} \mathrm{O}_{5}$ & 348.4393 & 1238.4378 & 1239.4458 & 620.2269 & 413.8206 & 310.61745 \\
\hline Hydroxylactam- $\mathrm{H}_{2} \mathrm{O}$ & $\mathrm{C}_{20} \mathrm{H}_{26} \mathrm{O}_{4}$ & 330.4240 & 1220.4225 & 1221.4305 & 611.21925 & 407.8155 & 306.11362 \\
\hline $\begin{array}{l}\text { Hydroxylactam- } \\
2 \mathrm{H}_{2} \mathrm{O}\end{array}$ & $\mathrm{C}_{20} \mathrm{H}_{24} \mathrm{O}_{3}$ & 312.4088 & 1202.4073 & 1203.4153 & 602.21165 & 401.81043 & 301.60982 \\
\hline Cross-link & $\mathrm{C}_{20} \mathrm{H}_{33} \mathrm{O}_{4}$ & 337.4796 & 2114.4526 & 2115.4606 & 1058.2343 & 705.82553 & 529.62115 \\
\hline Cross-link- $\mathrm{H}_{2} \mathrm{O}$ & $\mathrm{C}_{20} \mathrm{H}_{31} \mathrm{O}_{3}$ & 319.4643 & 2096.4373 & 2097.4453 & 1049.2266 & 699.82043 & 525.11732 \\
\hline Cross-link- $2 \mathrm{H}_{2} \mathrm{O}$ & $\mathrm{C}_{20} \mathrm{H}_{29} \mathrm{O}_{2}$ & 301.4491 & 2078.4221 & 2079.4301 & 1040.2190 & 693.81536 & 520.61352 \\
\hline Bis-pyrrole & $\mathrm{C}_{40} \mathrm{H}_{58} \mathrm{O}_{6}$ & 634.8969 & 2410.8619 & 2411.8699 & 1206.4389 & 804.62863 & 603.72347 \\
\hline Bis-pyrrole- $\mathrm{H}_{2} \mathrm{O}$ & $\mathrm{C}_{40} \mathrm{H}_{56} \mathrm{O}_{5}$ & 616.8816 & 2392.8466 & 2393.8546 & 1197.4313 & 798.62353 & 599.21965 \\
\hline Bis-pyrrole-2 $\mathrm{H}_{2} \mathrm{O}$ & $\mathrm{C}_{40} \mathrm{H}_{54} \mathrm{O}_{4}$ & 598.8664 & 2374.8314 & 2375.8394 & 1188.4237 & 792.61846 & 594.71585 \\
\hline Tris-pyrrole & $\mathrm{C}_{60} \mathrm{H}_{88} \mathrm{O}_{9}$ & 953.3353 & 3617.2829 & 3618.2909 & 1809.6495 & 1206.7690 & 905.3287 \\
\hline Tris-pyrrole- $\mathrm{H}_{2} \mathrm{O}$ & $\mathrm{C}_{60} \mathrm{H}_{86} \mathrm{O}_{8}$ & 935.3200 & 3599.2676 & 3600.2756 & 1800.6418 & 1200.7639 & 900.8249 \\
\hline Tris-pyrrole- $2 \mathrm{H}_{2} \mathrm{O}$ & $\mathrm{C}_{60} \mathrm{H}_{84} \mathrm{O}_{7}$ & 917.3047 & 3581.2523 & 3582.2603 & 1791.6342 & 1194.7588 & 896.3211 \\
\hline Tris-pyrrole- $3 \mathrm{H}_{2} \mathrm{O}$ & $\mathrm{C}_{60} \mathrm{H}_{82} \mathrm{O}_{6}$ & 899.2894 & 3563.2370 & 3564.2450 & 1782.6265 & 1188.7537 & 891.8173 \\
\hline Pyrrole+Lactam & $\mathrm{C}_{40} \mathrm{H}_{58} \mathrm{O}_{7}$ & 650.8963 & 2426.8613 & 2427.8693 & 1214.4387 & 809.9618 & 607.7233 \\
\hline $\mathrm{Pyr}+\mathrm{Lac}-\mathrm{H}_{2} \mathrm{O}$ & $\mathrm{C}_{40} \mathrm{H}_{56} \mathrm{O}_{6}$ & 632.8810 & 2408.8460 & 2409.8540 & 1205.4310 & 803.9567 & 603.2195 \\
\hline Pyr+Lac- $2 \mathrm{H}_{2} \mathrm{O}$ & $\mathrm{C}_{40} \mathrm{H}_{54} \mathrm{O}_{5}$ & 614.8658 & 2390.8307 & 2391.8387 & 1196.4234 & 797.9516 & 598.7157 \\
\hline
\end{tabular}

EVHHQKL (Monoisotopic mw):889.476977, (+1)890.454253, (+2)445.745765 H:1.0078 $\quad \mathrm{H}_{2} \mathrm{O}: 18.0106$

\begin{tabular}{|c|c|c|c|c|c|c|c|}
\hline Adducts & $\begin{array}{l}\text { Adduct } \\
\text { Formula }\end{array}$ & $\begin{array}{l}\text { Adduct } \\
\text { MW }\end{array}$ & $\begin{array}{l}\text { Product } \\
\text { MW }\end{array}$ & $\begin{array}{l}\text { Plus one } \\
\text { charge }(+1)\end{array}$ & $\begin{array}{l}\text { Plus two } \\
\text { charges }(+2)\end{array}$ & $\begin{array}{l}\text { Plus three } \\
\text { charges }(+3)\end{array}$ & $\begin{array}{l}\text { Plus four } \\
\text { charges }(+4)\end{array}$ \\
\hline Pyrrole & $\mathrm{C}_{20} \mathrm{H}_{28} \mathrm{O}_{3}$ & 316.2038 & 1205.6808 & 1206.6886 & 603.8482 & 402.9014 & 302.4280 \\
\hline Pyrrole $-\mathrm{H}_{2} \mathrm{O}$ & $\mathrm{C}_{20} \mathrm{H}_{26} \mathrm{O}_{2}$ & 298.1933 & 1187.6703 & 1188.6781 & 594.84295 & 396.8979 & 297.9254 \\
\hline Lactam & $\mathrm{C}_{20} \mathrm{H}_{28} \mathrm{O}_{4}$ & 332.1988 & 1221.6750 & 1222.6828 & 611.8453 & 408.2328 & 306.4266 \\
\hline Lactam- $\mathrm{H}_{2} \mathrm{O}$ & $\mathrm{C}_{20} \mathrm{H}_{26} \mathrm{O}_{3}$ & 314.1882 & 1203.6652 & 1204.6730 & 602.8404 & 402.2295 & 301.9241 \\
\hline Hydroxylactam & $\mathrm{C}_{20} \mathrm{H}_{28} \mathrm{O}_{5}$ & 348.1937 & 1237.6707 & 1238.6785 & 619.84315 & 413.5647 & 310.4255 \\
\hline Hydroxylactam- $\mathrm{H}_{2} \mathrm{O}$ & $\mathrm{C}_{20} \mathrm{H}_{26} \mathrm{O}_{4}$ & 330.1831 & 1219.6601 & 1220.6679 & 610.83785 & 407.5612 & 305.9228 \\
\hline $\begin{array}{l}\text { Hydroxylactam- } \\
2 \mathrm{H}_{2} \mathrm{O}\end{array}$ & $\mathrm{C}_{20} \mathrm{H}_{24} \mathrm{O}_{3}$ & 312.1725 & 1201.6495 & 1202.6573 & 601.83255 & 401.5576 & 301.4202 \\
\hline Cross-link & $\mathrm{C}_{20} \mathrm{H}_{33} \mathrm{O}_{4}$ & 337.2379 & 2113.1685 & 2114.1763 & 1057.5921 & 705.3973 & 529.2999 \\
\hline Cross-link- $\mathrm{H}_{2} \mathrm{O}$ & $\mathrm{C}_{20} \mathrm{H}_{31} \mathrm{O}_{3}$ & 319.2273 & 2095.1579 & 2096.1657 & 1048.5867 & 699.3938 & 524.7973 \\
\hline Cross-link- $2 \mathrm{H}_{2} \mathrm{O}$ & $\mathrm{C}_{20} \mathrm{H}_{29} \mathrm{O}_{2}$ & 301.2168 & 2077.1473 & 2078.1551 & 1039.5814 & 693.39023 & 520.2946 \\
\hline Bis-pyrrole & $\mathrm{C}_{40} \mathrm{H}_{58} \mathrm{O}_{6}$ & 634.4233 & 2409.3461 & 2410.3539 & 1205.6808 & 804.12316 & 603.3443 \\
\hline Bis-pyrrole- $\mathrm{H}_{2} \mathrm{O}$ & $\mathrm{C}_{40} \mathrm{H}_{56} \mathrm{O}_{5}$ & 616.4128 & 2391.3355 & 2392.3433 & 1196.6755 & 798.11963 & 598.8417 \\
\hline Bis-pyrrole- $2 \mathrm{H}_{2} \mathrm{O}$ & $\mathrm{C}_{40} \mathrm{H}_{54} \mathrm{O}_{4}$ & 598.4022 & 2373.3249 & 2374.3327 & 1187.6702 & 792.1161 & 594.3390 \\
\hline Tris-pyrrole & $\mathrm{C}_{60} \mathrm{H}_{88} \mathrm{O}_{9}$ & 952.6428 & 3617.9588 & 3618.9666 & 1809.9872 & 1206.9941 & 905.4975 \\
\hline Tris-pyrrole- $\mathrm{H}_{2} \mathrm{O}$ & $\mathrm{C}_{60} \mathrm{H}_{86} \mathrm{O}_{8}$ & 934.6322 & 3599.9482 & 3600.9560 & 1800.9819 & 1200.9905 & 900.9948 \\
\hline Tris-pyrrole- $2 \mathrm{H}_{2} \mathrm{O}$ & $\mathrm{C}_{60} \mathrm{H}_{84} \mathrm{O}_{7}$ & 916.6216 & 3581.9376 & 3582.9454 & 1791.9766 & 1194.9870 & 896.4922 \\
\hline Tris-pyrrole- $3 \mathrm{H}_{2} \mathrm{O}$ & $\mathrm{C}_{60} \mathrm{H}_{82} \mathrm{O}_{6}$ & 898.6110 & 3563.9270 & 3564.9348 & 1782.9713 & 1188.9835 & 891.9895 \\
\hline Pyrrole+Lactam & $\mathrm{C}_{40} \mathrm{H}_{58} \mathrm{O}_{7}$ & 650.4183 & 2425.3411 & 2426.3489 & 1213.6783 & 809.4548 & 607.3431 \\
\hline Pyr+Lac- $\mathrm{H}_{2} \mathrm{O}$ & $\mathrm{C}_{40} \mathrm{H}_{56} \mathrm{O}_{6}$ & 632.4077 & 2407.3305 & 2408.3383 & 1204.6730 & 803.4513 & 602.8404 \\
\hline $\mathrm{Pyr}+\mathrm{Lac}-2 \mathrm{H}_{2} \mathrm{O}$ & $\mathrm{C}_{40} \mathrm{H}_{54} \mathrm{O}_{5}$ & 614.3971 & 2389.3209 & 2390.3287 & 1195.6682 & 797.4481 & 598.3380 \\
\hline
\end{tabular}


Figure S1. N-Acetyl-Gly-Lys-O-Methyl ester reactions with iso[4] $\mathrm{LGE}_{2}$ in different molar ratio and time points.

(1) N-Acetyl-Gly-Lys-O-Methyl ester reacts with iso[4]LGE 2 (Dipeptide: Iso4 = 2000:1 molar ratio) in $50 \mathrm{mM}$ NEM OAc $\mathrm{pH} 8.6$ for 1 day, 4 days, 8 days, 15 days, 21 days and 50 days.
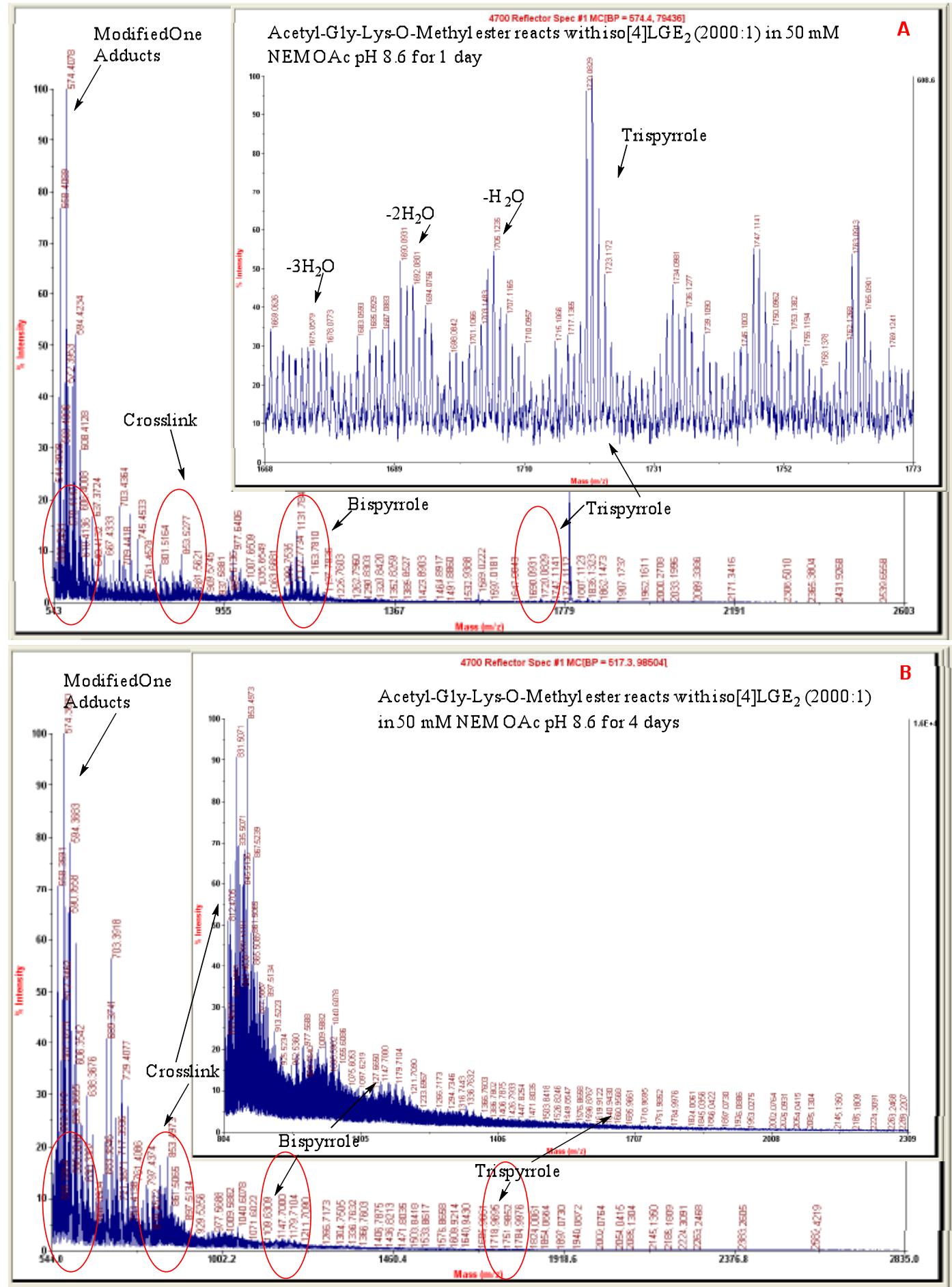

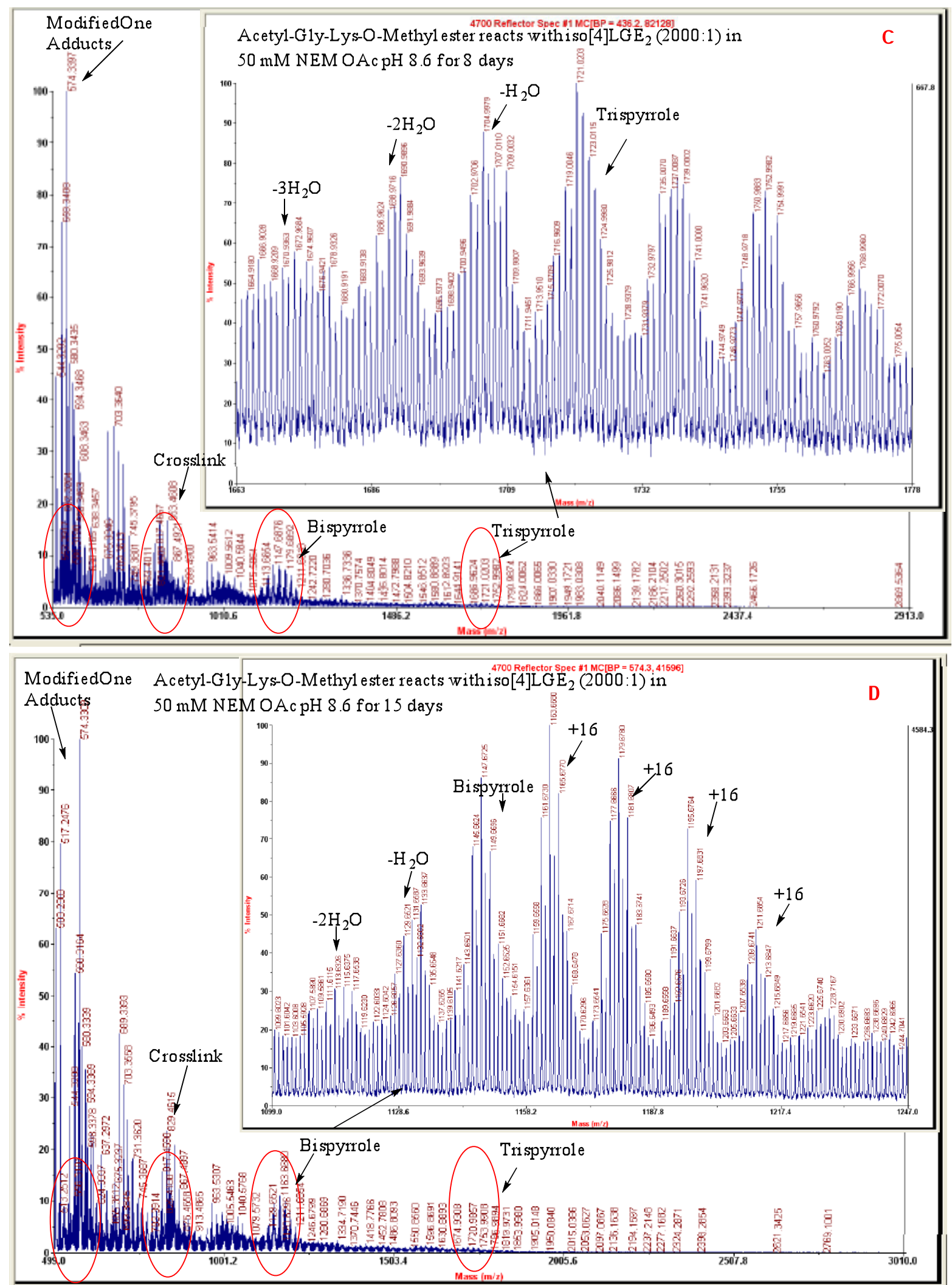




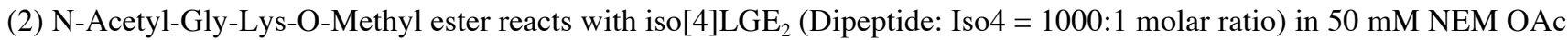
pH 8.6 for 1 day, 4 days, 8 days, 15 days, 21 days and 50 days.


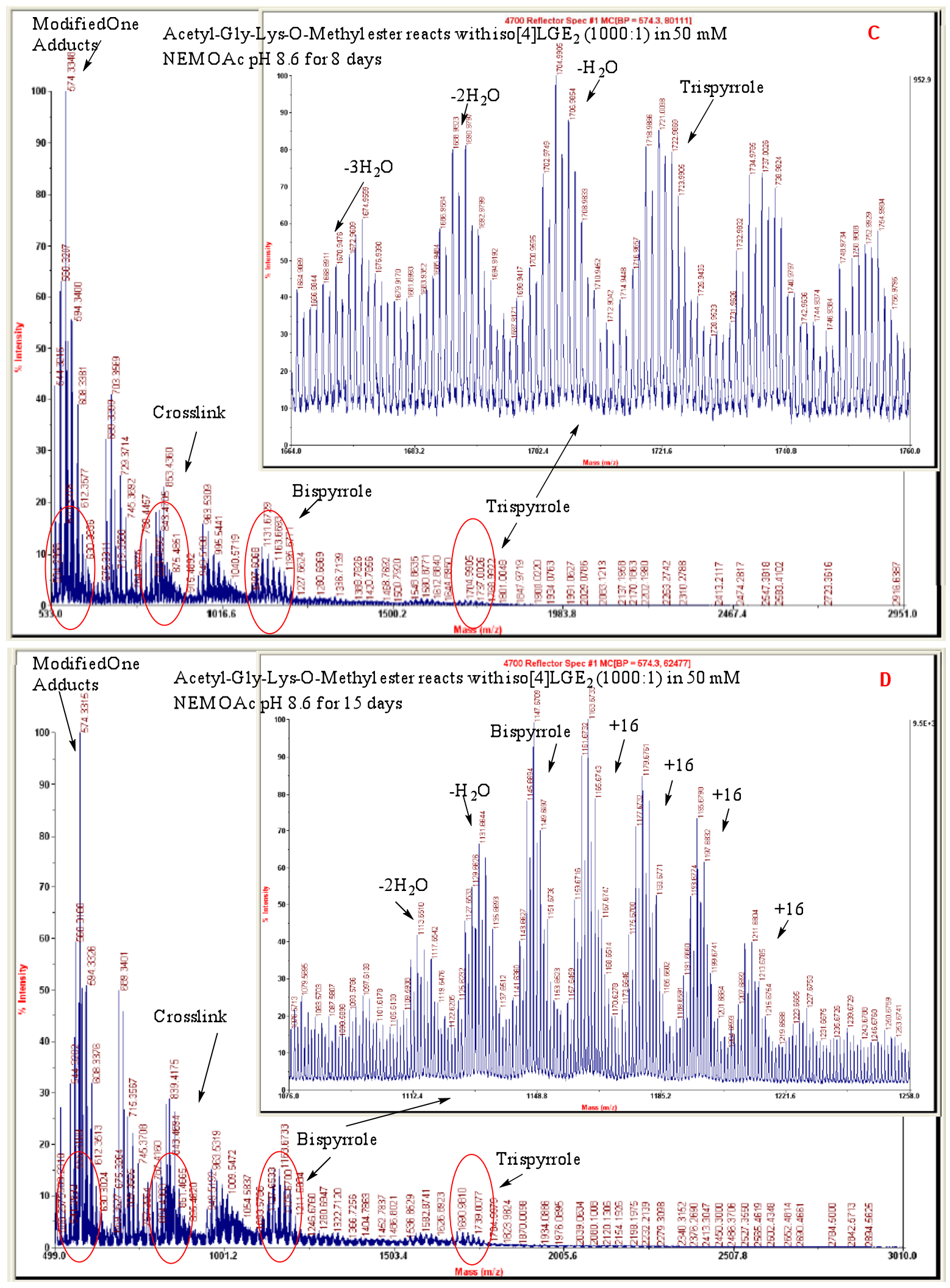

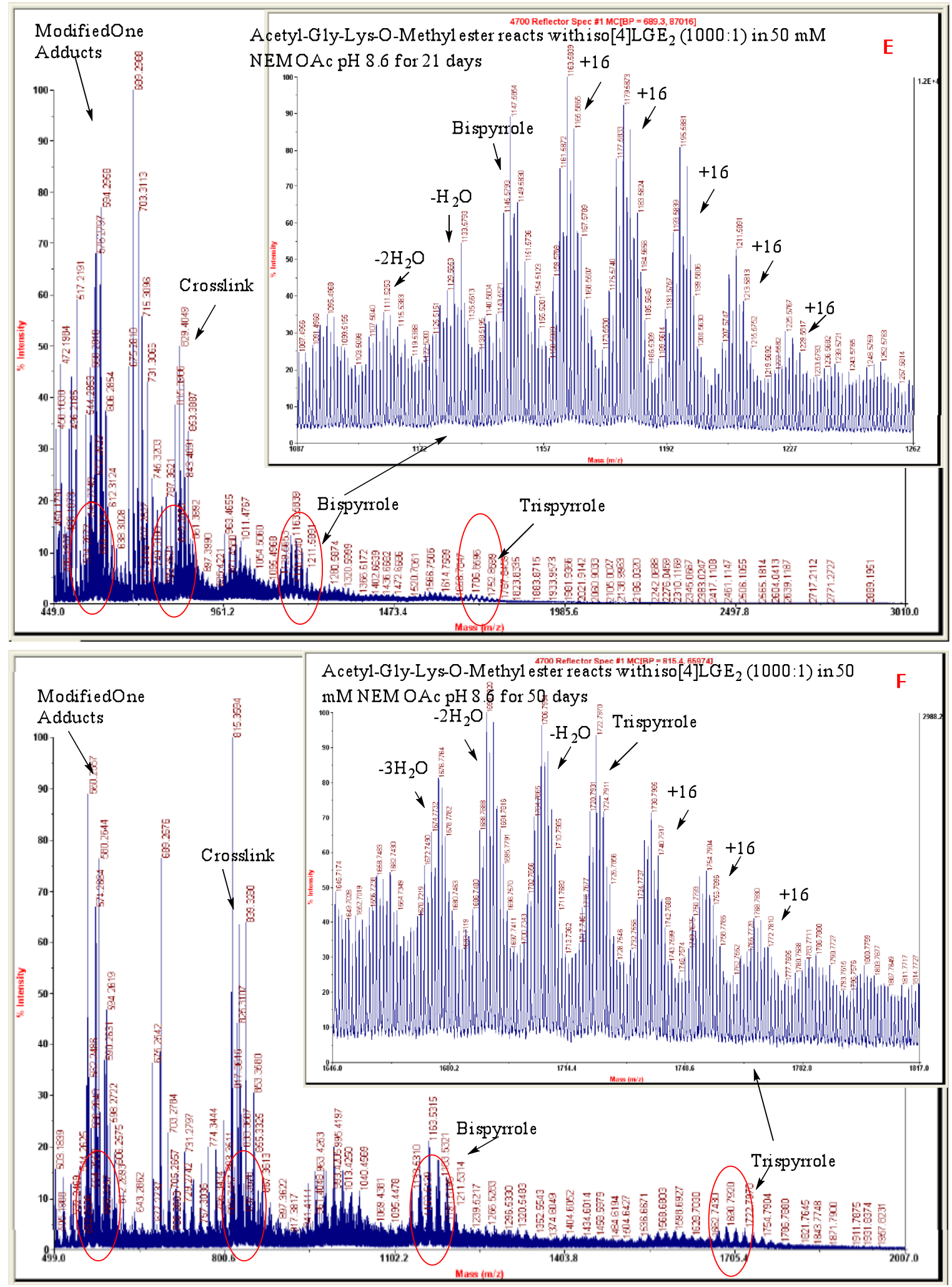
(3) N-Acetyl-Gly-Lys-O-Methyl ester reacts with iso[4]LGE 2 (Dipeptide: Iso4 = 200:1 molar ratio) in $50 \mathrm{mM}$ NEM OAc $\mathrm{pH} 8.6$ for 1 day, 4 days, 8 days, 15 days, 21 days and 50 days.


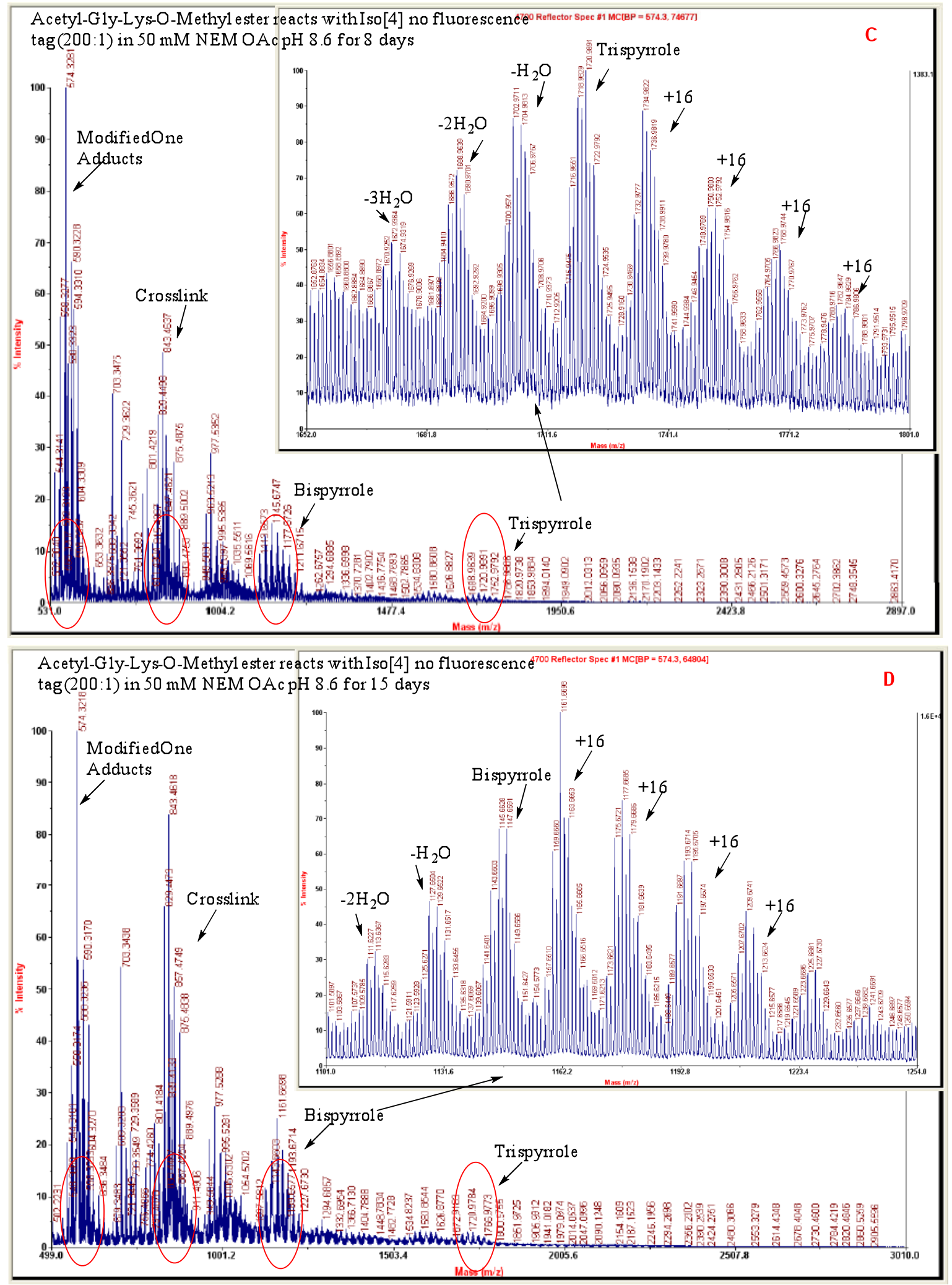



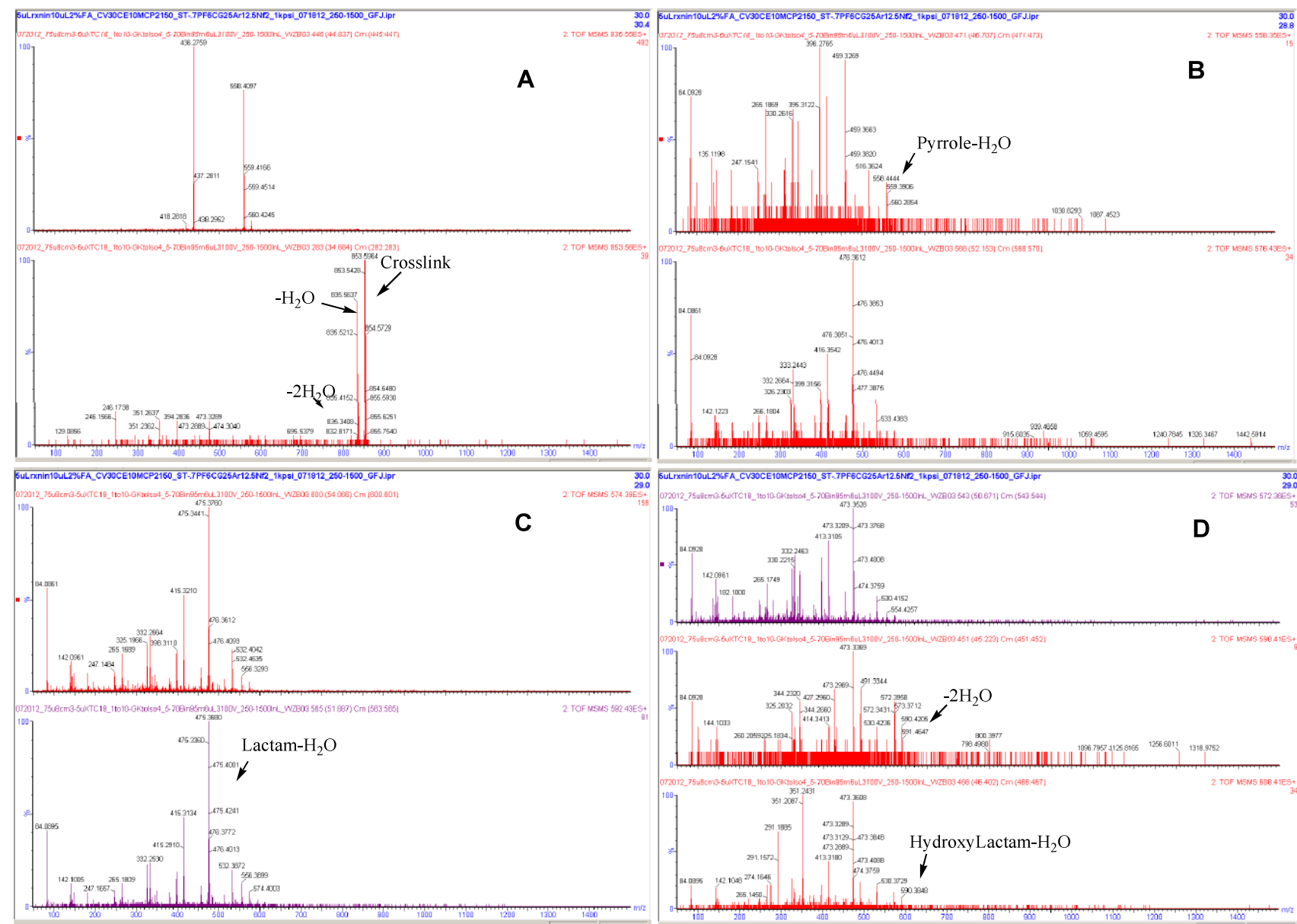

Figure S2. ESI-MS/MS of molecular ions at A) aminal cross-link, B) pyrrole, C) lactam and D) hydroxylactam corresponding to an iso[4] $\mathrm{LGE}_{2}-(\mathrm{N}-$ Acetyl-Gly-Lys-OMe) adduct. 

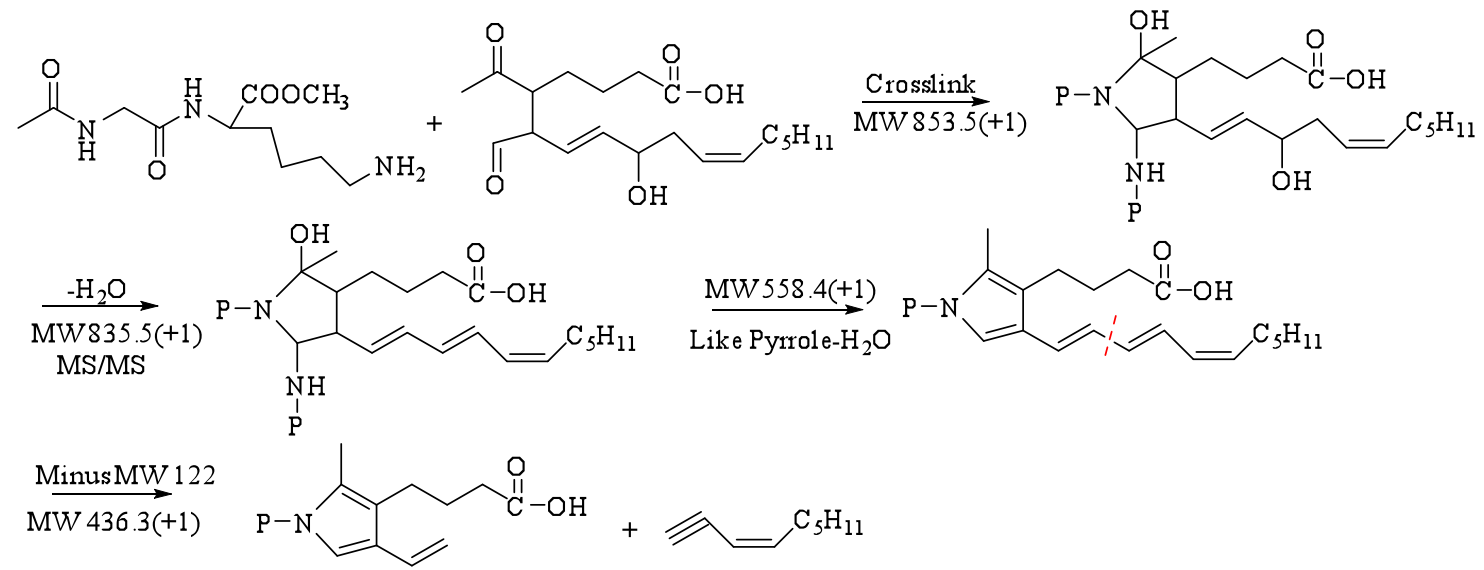



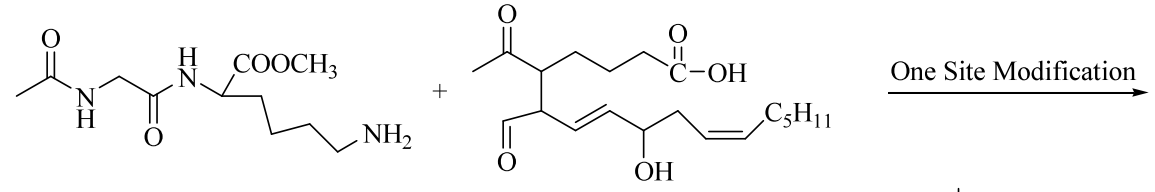

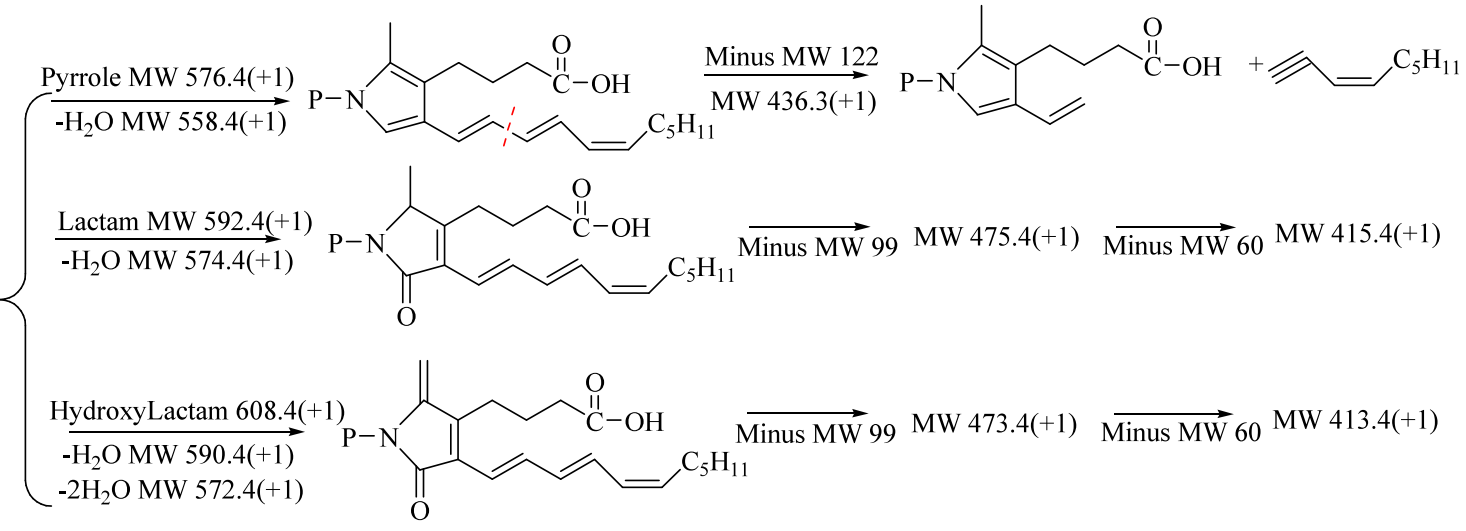

Scheme S1. Possible fragmentation of (N-Acetyl-Gly-Lys-OMe)-iso[4] $\mathrm{LGE}_{2}$ adducts. 
Figure S3. Amyloid (11-17) EVHHQKL peptide reactions with iso[4] $\mathrm{LGE}_{2}$ in different molar ratio and time points.

(1) Amyloid (11-17) "EVHHQKL" reacts with iso[4]LGE 2 (Peptide: Iso4 = 2000:1) in $50 \mathrm{mM}$ NEM OAc pH 8.6 for 1 day, 4 days, 15 days, 21 days and 50 days.
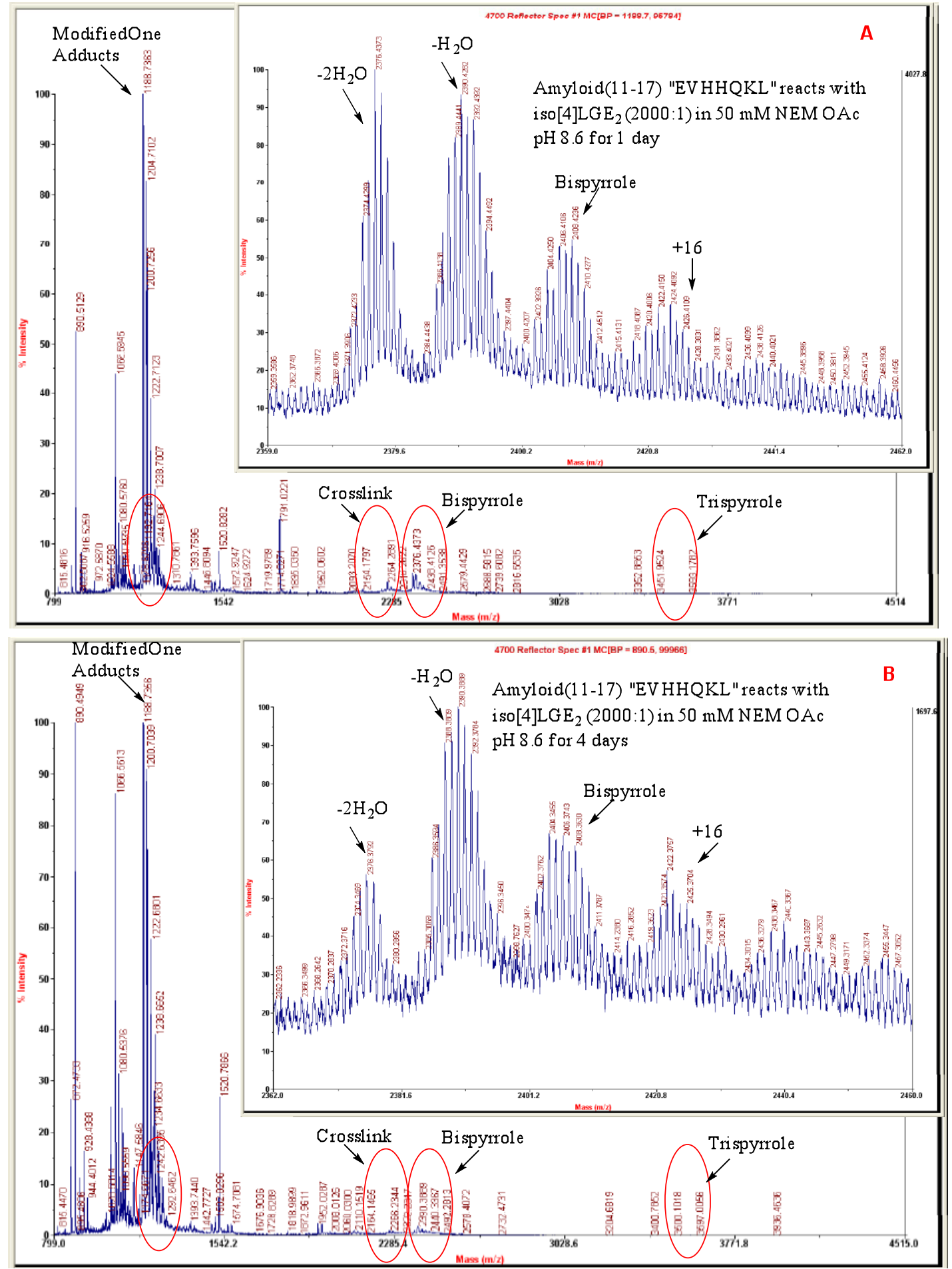




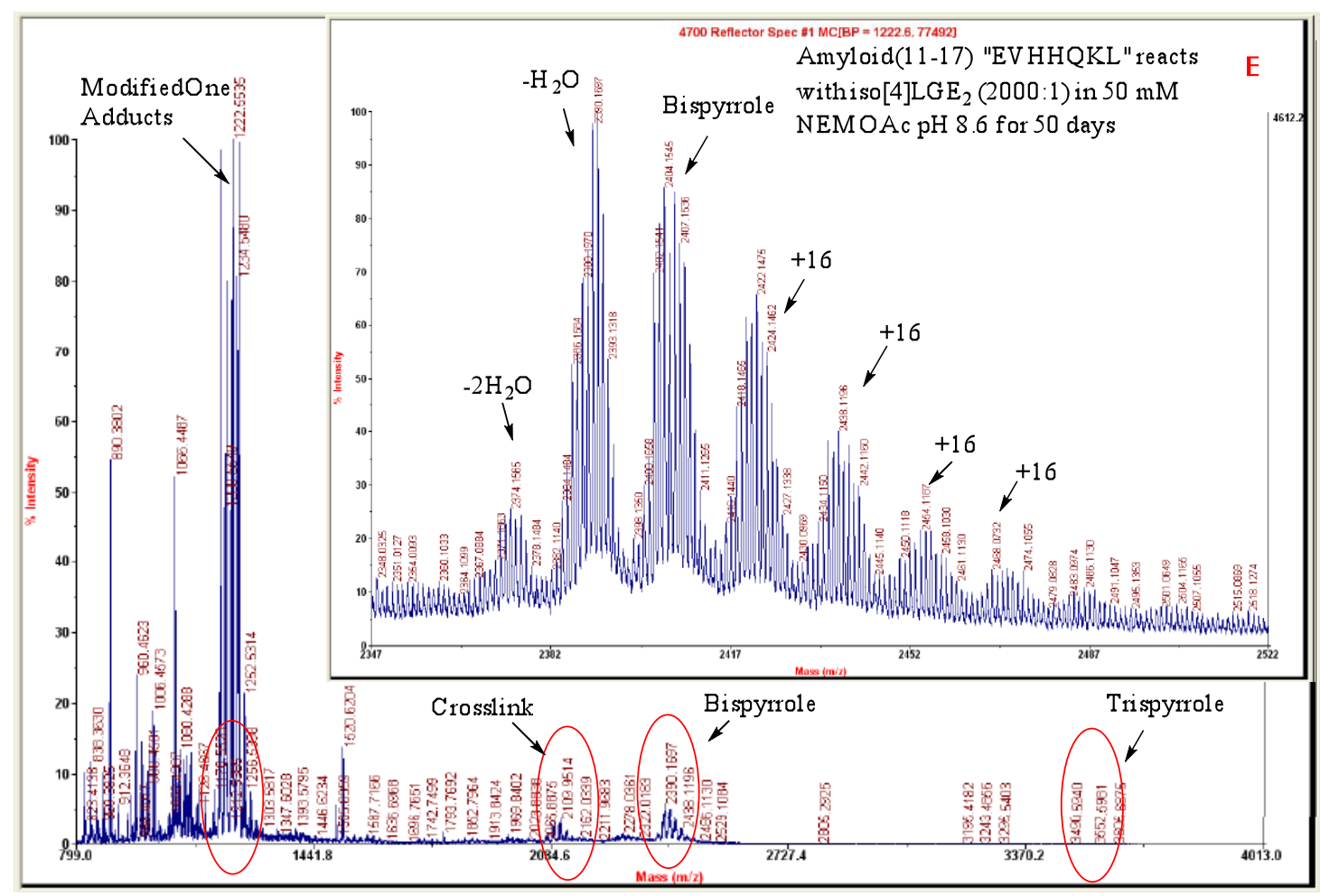

17) "EVHHQKL" reacts with iso[4]LGE (Peptide: Iso4 = 200:1) in $50 \mathrm{mM} \mathrm{NEM} \mathrm{OAc} \mathrm{pH} 8.6$ for 1 day, 4 days, 7 days, 15 days, 21 days and 50 days.

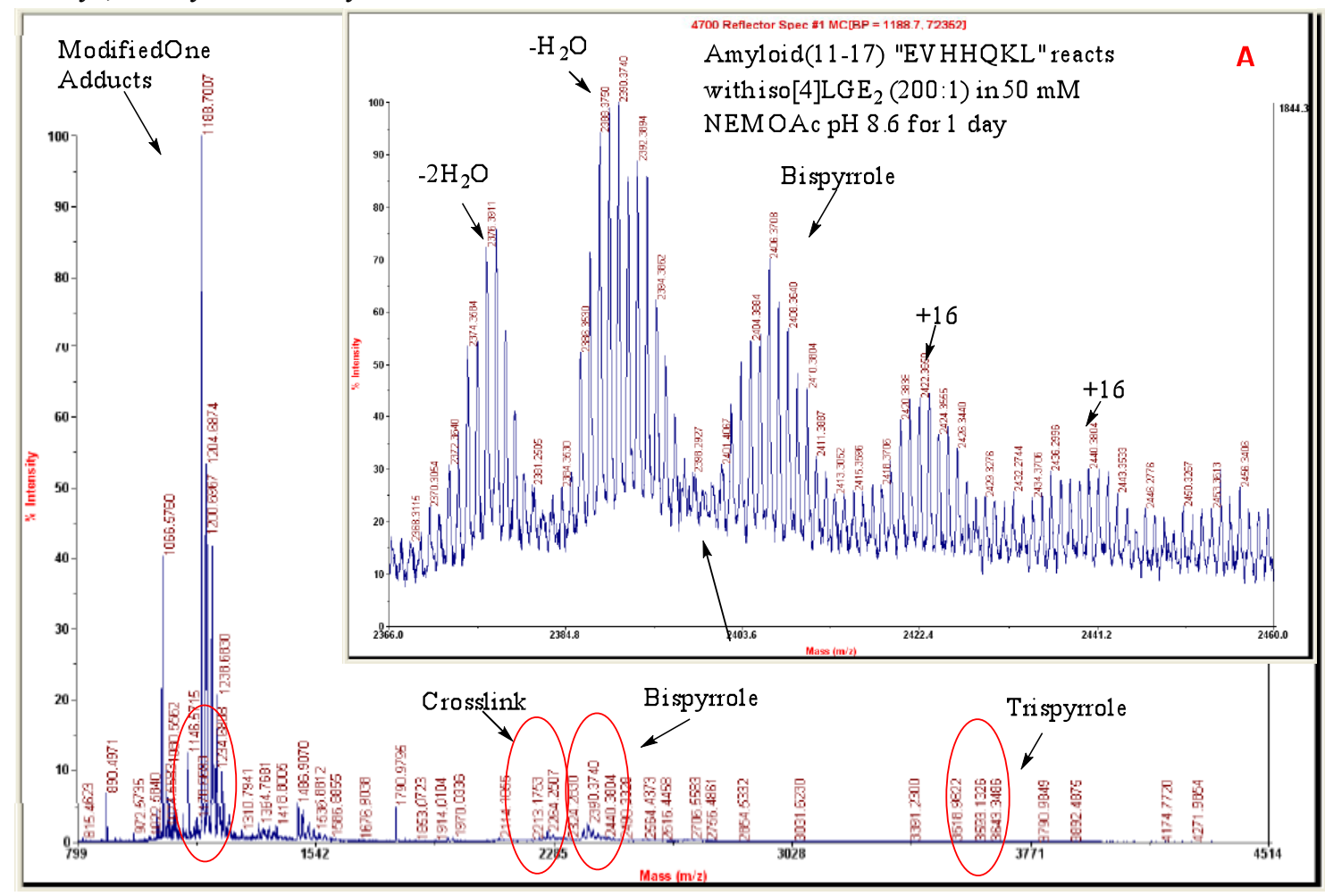



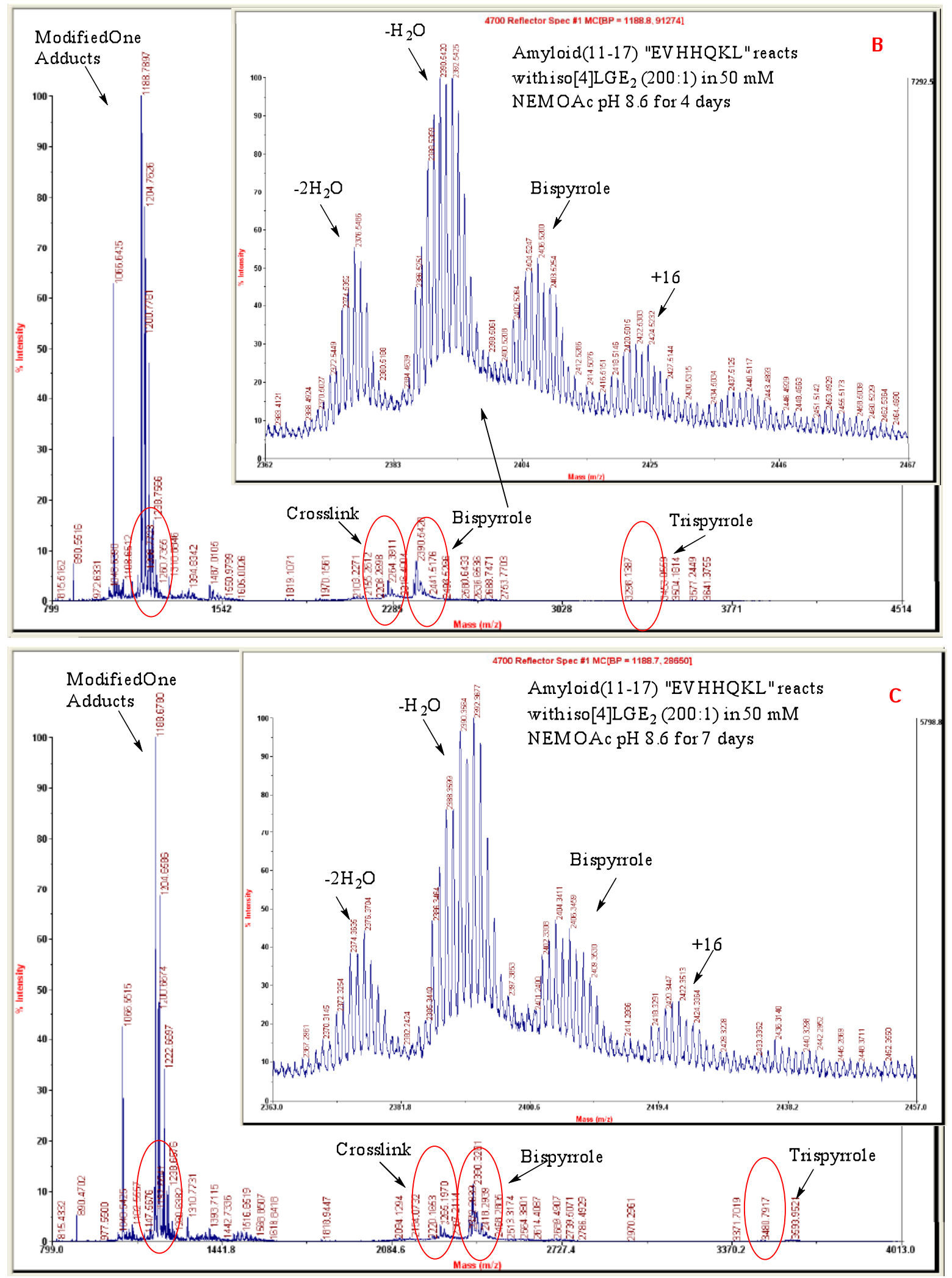






